\title{
EMBEDDING OF CLOSED CATEGORIES INTO MONOIDAL CLOSED CATEGORIES
}

\author{
BY
}

\author{
MIGUEL L. LAPLAZA
}

\begin{abstract}
S. Eilenberg and G. M. Kelly have defined a closed category as a category with internal homomorphism functor, left Yoneda natural arrows, unity object and suitable coherence axioms. A monoidal closed category is a closed category with an associative tensor product which is adjoint to the int-hom. This paper proves that a closed category can be embedded in a monoidal closed category: the embedding preserves any associative tensor product which may exist. Besides the usual tools of the theory of closed categories the proof uses the results of B. Day on promonoidal structures.
\end{abstract}

Introduction. The concept of closed category has been extensively developed in [6, 424-471] and involves basically a category $V$, an internal homomorphism functor [ ]: $\mathbf{V}^{\text {op }} \times \mathbf{V} \rightarrow \mathbf{V}$ and natural arrows of Yoneda type, $\left.L:\left[\begin{array}{ll}B & C\end{array}\right] \rightarrow\left[\begin{array}{ll}A & B\end{array}\right]\left[\begin{array}{ll}A & C\end{array}\right]\right]$. If there is also a tensor product with $-\otimes B$ left adjoint to $[B-]$ we have a monoidal closed category [6, 471-548], [7]. A biclosed category is a monoidal closed category with an additional int-hom, \{\} , such that $B \otimes-$ is left adjoint to $\{B-\}$. Within the framework of the monoidal closed categories the "psychological dual" of a closed category is a monoidal category [1], [8], [6] whose structure reduces to the tensor product.

The object of this paper is to prove that any closed category can be embedded structure-preservingly in a biclosed category. Moreover this embedding preserves any associative tensor product which may exist: we speak of preservation up to isomorphisms compatible with the structure under consideration.

Our basic tool is the concept of promonoidal structure of B. Day [2], [3] and the calculations require a frequent use of the representation theorem for closed categories [4, Theorem I.8.6, 457]. The argument goes as follows: For $V$ a closed category the map $A m[A-]$ defines a functor from $V^{\circ}$ into the category $\mathbf{E}$ of the $\mathbf{V}$-endofunctors of $\mathbf{V}$ and $\mathbf{V}$-natural transformations. The category $\mathbf{E}$ is monoidal for the composition of endofunctors and by [2], [3] induces a biclosed monoidal structure on the category, [E, S], of set-valued

Received by the editors October 20, 1975.

AMS (MOS) subject classifications (1970). Primary 18D15; Secondary 18B15, 18D10.

Key words and phrases. Closed category, monoidal category, monoidal closed category, monoidal biclosed category, internal homomorphism functor, tensor product, promonoidal structure.

- American Mathematical Society 1977 
functors and natural transformations: our embedding is the composition of the functor from $\mathbf{V}$ into $\mathbf{E}$ with the Yoneda embedding of $\mathbf{E}$ into [E, S]. We will omit the detail of the computations: they are straightforward consequences of [4] and [2] or [3].

The author is deeply indebted to B. Day who in several informal conversations in the University of Chicago during the Summer of 1972 pointed out the possibility of using the promonoidal structures in this type of problems, to the University of Chicago for its hospitality during that period, to Professor S. Mac Lane for his advice in the development of the ideas contained in this work and to Professor G. M. Kelly who localized several errors in a previous version of the paper and suggested improvements.

For all undefined concepts we refer to [9] and throughout the paper we will adopt the following conventions:

(a) To omit as many subindices as can be recovered from the context.

(b) To use the same symbol to denote an arrow or natural transformation and its inverse.

(c) The symbol $[A, B]$ or $[A B]$ will represent the action of the int-hom and for $r>2,\left[A_{1} \cdots A_{r}\right]$ is defined recurrently by $\left[A_{1} \cdots A_{r}\right]=$ $\left[A_{1}\left[A_{2} \cdots A_{r}\right]\right]$.

(d) The term "natural transformation" is used in the general sense of [5] and includes the concept of diagonal naturality of [9, 214-218].

1. Basic definitions. A closed category is a category $\mathbf{V}$ with the following structure:

(1) A functor, [ ]: $\mathbf{V}^{\text {op }} \times \mathbf{V} \rightarrow \mathbf{V}$.

(2) A fixed object $I$ of $\mathbf{V}$.

(3) A natural isomorphism, $i_{A}: A \rightarrow[I A]$.

(4) Natural transformations,

$$
j_{A}: I \rightarrow[A A], \quad L_{A, B, C}:\left[\begin{array}{ll}
B C & \rightarrow
\end{array}\left[\left[\begin{array}{ll}
A & B
\end{array}\right]\left[\begin{array}{ll}
A & C
\end{array}\right]\right] .\right.
$$

This structure satisfies the axioms CCl-CC4 of $[4,428-429]$ and

CC 5. The natural map from $\mathrm{V}(A, B)$ into $\mathrm{V}(I,[A B])$ defined by

$$
f: A \rightarrow B \rightsquigarrow[1 f] j: I \rightarrow[A A] \rightarrow[A B],
$$

is an isomorphism.

This definition is basically the original concept of closed category of Eilenberg-Kelly $[4,428]$.

A consequence of the above axiom is the relation

$$
i_{I}=j_{I}: I \rightarrow[I I] \text {. }
$$

If $\mathbf{V}$ and $\mathbf{W}$ are closed categories a closed embedding of $\mathbf{V}$ into $\mathbf{W}$ is a closed functor $\left(F, F_{A, B}, F_{1}\right)[4,433]$ such that $F$ is full and faithful and $F_{A, B}$ and $F_{I}$ are isomorphisms. Intuitively speaking a closed embedding is an 
embedding which preserves the closed structure up to suitable isomorphisms.

From now on $\mathbf{V}$ will denote a closed category and we will prove that there is a closed embedding of $\mathbf{V}$ into a monoidal closed category. In fact we will construct an embedding which preserves strictly the closed structure of $\mathbf{V}$.

2. The embedding. The category $\mathbf{E}$ of $\mathbf{V}$-endofunctors of $\mathbf{V}$ and $\mathbf{V}$-natural transformations $[4,444,445]$ is a monoidal category with the composition of endofunctors for tensor product. We will denote $\mathbf{B}=[\mathbf{E}, \mathbf{S}]$ the category of set-valued functors with domain $\mathbf{E}$ and natural transformations. According to Day's results [2], [3], the monoidal structure of $\mathbf{E}$ induces a monoidal closed structure on $\mathbf{B}$ given by

$$
\begin{aligned}
(R \otimes S)(-) & =\int^{X, Y} R(X) \times S(Y) \times \mathbf{E}(X \otimes Y,-), \\
{[R S](-) } & =\int_{X} \mathbf{S}(R(X), S(-\otimes X)) .
\end{aligned}
$$

Moreover B is a biclosed monoidal category but we will not use this part of its structure. The above definition determines the structure of B up to isomorphisms and we will select later the most convenient representatives of its elements.

The associativity of the tensor product of $\mathbf{B}$ is defined via coprojections of coends by the commutativity of the diagram:

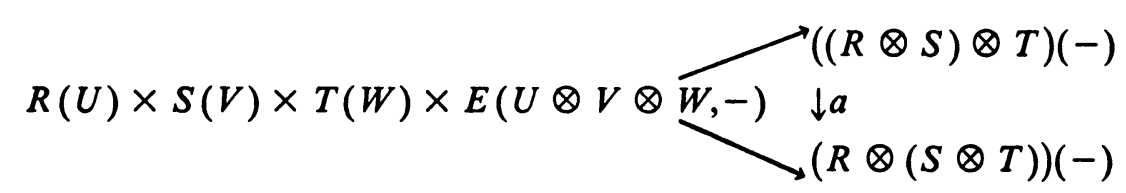

We take the unity object of $B$ to be the functor

$$
q_{I}(-)=\mathrm{V}(I,-(I))
$$

and the natural arrow $l: R \rightarrow q_{I} \otimes R$ is defined by the composite

$$
\text { uv: } R(F) \rightarrow V(I,[I I]) \times R(F) \times \mathbf{E}([I, F(-)], F(-)) \rightarrow\left(q_{I} \otimes R\right)(F) \text {, }
$$

where $u$ is the coprojection of the coend $q_{I} \otimes R$ and $v$ is given by $v(x)=$ $\left(j_{I}, x, i_{F}\right)$. Analogously the natural arrow $r: R \rightarrow R \otimes q_{I}$ is defined by the composite

$$
\text { uv: } R(F) \rightarrow R(F) \times \mathrm{V}(I,[I I]) \otimes \mathrm{E}(F[I-], F(-)) \rightarrow\left(R \otimes q_{I}\right)(F),
$$

where $v$ is defined by $v(x)=\left(x, j_{l}, F i\right)$ and $u$ is a coprojection of the coend $R \otimes q_{I}$.

The natural arrow $e:[S T] \otimes S \rightarrow T$ of $\mathbf{B}$ is determined by the fact of being its composition with the $\{X, Y\}$-coprojection of $[S T] \otimes S$ the arrow 


$$
\begin{aligned}
u e(v \times 1 \times 1): & {[S T](X) \times S(Y) \times \mathbf{E}(X \otimes Y, F) } \\
\rightarrow & \mathbf{S}(S(Y), T(X \otimes Y)) \times S(Y) \times \mathbf{E}(X \otimes Y, F) \\
\rightarrow & T(X \otimes Y) \times \mathbf{E}(X \otimes Y, F) \rightarrow T(F),
\end{aligned}
$$

where $v$ is a projection of the end [ST], $e$ is the evaluation of $\mathbf{S}$ and $u$ is defined by $u(x, f)=T(f)(x)$.

The natural arrow $d: R \rightarrow[S, R \otimes S]$ of $\mathrm{B}$ is determined by the fact of being its composition with the $Y$-projection of the end $[S, R \otimes S]$ the arrow

$$
\begin{aligned}
\mathbf{S}(1, u) \mathbf{S}(1, v) d: & R(F) \rightarrow \mathbf{S}(S(Y), R(F) \times S(Y)) \\
& \rightarrow \mathbf{S}(S(Y), R(F) \times S(Y) \times E(F \otimes Y, F \otimes Y)) \\
& \rightarrow \mathbf{S}(S(Y),(R \otimes S)(F \otimes Y)),
\end{aligned}
$$

where $d$ is the natural arrow of the monoidal closed structure of $\mathbf{S}, \boldsymbol{u}$ is the $\{F, Y\}$-coprojection of the coend $R \otimes S$ and $v$ is defined by $v(x, y)=$ $(x, y, 1)$.

We have full and faithful contravariant functors,

$$
p: \mathbf{V} \rightarrow \mathbf{E}, y: \mathbf{E} \rightarrow[\mathbf{E}, \mathbf{S}]=\mathbf{B},
$$

where $y$ is the Yoneda embedding and $p$ is defined by $p(A)=[A-]$. The functor $q=y p$ is a covariant full and faithful functor which can be defined up to an isomorphism by

$$
q: A m q_{A}(-)=\mathrm{V}(I,-(A)) \text {. }
$$

We will show that $q$ is a closed functor by fixing the monoidal closed structure of $\mathbf{B}$ in such a way that $q$ preserves strictly the closed structure of $\mathbf{V}$. After our selection of the unity object of $\mathbf{B}$ it is clear that $q$ preserves the unity object.

Proposition 1. The functor $q$ preserves the int-hom.

Proof. For any objects $A$ and $B$ of $\mathbf{V}$,

$$
\begin{aligned}
{\left[q_{A} q_{B}\right](F) } & =\int_{X} \mathbf{S}\left(q_{A}(X), q_{B}(F \otimes X)\right) \\
& =\int_{X} \mathbf{S}(\mathbf{E}([A-], X), \mathbf{V}(I,(F \otimes X)(B))) \\
& =\mathbf{V}(I, F[A B])=q_{[A B]}(F) .
\end{aligned}
$$

For the next propositions we have to detail the structure of $\mathbf{B}$ when the involved elements are in the image of $q$. By convenient choices and straightforward computations we can prove the following facts:

(i) $\left(q_{A} \otimes q_{B}\right)(F)=\mathbf{E}([A, B,-], F)$. 
(ii) $\left(\left(q_{A} \otimes q_{B}\right) \otimes q_{C}\right)(F)=\left(q_{A} \otimes\left(q_{B} \otimes q_{C}\right)\right)(F)=\mathbf{E}([A, B, C,-], F)$.

(iii) $q_{I} \otimes q_{A}=q_{A} \otimes q_{I}=q_{A}$.

(iv) $\left.\left(\left[q_{A} q_{B}\right] \otimes q_{A}\right)(F)=\mathrm{E}\left(\left[\begin{array}{ll}A & B\end{array}\right], A,-\right], F\right)$.

(v) The arrow $e:\left[q_{A} q_{B}\right] \otimes q_{A} \rightarrow q_{B}$ is defined by

$$
f:[[A B], A,-] \rightarrow F \rightsquigarrow f_{B} j: I \rightarrow[[A B], A, B] \rightarrow F B .
$$

(vi) $\left[q_{B}, q_{A} \otimes q_{C}\right](F)=\mathrm{E}([A, C,-], F[B-])$.

(vii) The arrow $d: q_{A} \rightarrow\left[q_{B}, q_{A} \otimes q_{B}\right]$ is defined by

$$
\begin{aligned}
f: I & \rightarrow F(A) \leftrightarrow i[f 1] F:[A, B,-] \rightarrow[F A, F[B-]] \\
& \rightarrow[I, F[B-]] \rightarrow F[B-] .
\end{aligned}
$$

Proposition 2. The functor $q$ preserves $j$ and $i$.

Proof. It reduces to calculating the composites,

$$
\begin{aligned}
& j=[1 r] d: q_{I} \rightarrow\left[q_{A}, q_{I} \otimes q_{A}\right] \rightarrow\left[q_{A} q_{A}\right], \\
& i=[1 l] d: q_{A} \rightarrow\left[q_{I}, q_{A} \otimes q_{I}\right] \rightarrow\left[q_{I} q_{A}\right] .
\end{aligned}
$$

Proposition 3. The functor $q$ preserves $L$.

PROOF. It is done in several steps. The first one is the calculation of the arrow

$$
t_{1}=e(1 \otimes e): q_{[B C]} \otimes q_{[A B]} \otimes q_{A} \rightarrow q_{[B C]} \otimes q_{B} \rightarrow q_{C},
$$

where

$$
q_{[B C]} \otimes q_{[A B]} \otimes q_{A}=\mathrm{E}([[B C],[A B], A,-], F) .
$$

It is easy to check that $t_{1}$ takes

$$
f:\left[\left[\begin{array}{ll}
B & C
\end{array}\right],\left[\begin{array}{ll}
A & B
\end{array}\right], A,-\right] \rightarrow F
$$

onto

$$
f c[1 \quad L] j: I \rightarrow[[B C], B, C] \rightarrow[[B C],[A B], A, C] \rightarrow F(C) .
$$

The second step is the calculation of the composite

$$
\begin{aligned}
t_{2} & =\left[1 t_{1}\right] d: q_{[B C]} \otimes q_{[A B]} \rightarrow\left[q_{A}, q_{[B C]} \otimes q_{[A B]} \otimes q_{A}\right] \\
& \left.\rightarrow\left[q_{A} q_{C}\right]=q_{[A} C\right],
\end{aligned}
$$

and it turns out that $t_{2}$ takes the arrow

$$
f:\left[\left[\begin{array}{ll}
B & C
\end{array}\right],\left[\begin{array}{ll}
A & B
\end{array}\right],-\right] \rightarrow F
$$

onto the arrow

$$
f_{(A C)}\left[\begin{array}{ll}
1 & L
\end{array}\right] j: I \rightarrow[[B C], B, C] \rightarrow[[B C],[A B], A, C] \rightarrow F[A C] .
$$

Finally we can compute $L$ as the composite 


$$
\begin{aligned}
& L=\left[1 t_{2}\right] d: q_{(B C]} \rightarrow\left[q_{(A B)}, q_{(B C]} \otimes q_{(A B)}\right] \\
& \left.\left.\rightarrow\left[q_{[A B}\right], q_{[A C]}\right]=q\left[{ }_{A B}\right], A, C\right],
\end{aligned}
$$

and it is easy to check that $L=q_{L}$ and the proposition is proved.

After this proposition we have proved that the functor $q$ is a closed embedding.

3. The preservation of the monoidal closed structure of $\mathbf{V}$. We will suppose from now on that $\mathbf{V}$ has a tensor product which extends the closed structure of $\mathbf{V}$ to a monoidal closed structure. We are going to prove that after redefining the tensor product of $\mathbf{B}$ the functor $q$ preserves also this monoidal closed structure.

For objects $A, B$ of $\mathbf{V}$ we have defined

$$
\left(q_{A} \otimes q_{B}\right)(F)=\mathbf{E}([A, B,-], F)
$$

and by using the monoidal closed structure of $\mathbf{V}$ we have the relation,

$$
\mathbf{E}([A, B,-], F) \approx \mathbf{E}([A \otimes B,-], F) \simeq \mathrm{V}(I, F(A \otimes B))=q_{A \otimes B}(F) .
$$

Therefore we can redefine

$$
q_{A} \otimes q_{B}=\mathrm{V}(I,-(A \otimes B))=q_{A B B}
$$

so that the functor $q$ preserves the tensor product of $\mathbf{V}$. It is very easy to check that $q$ preserves also the natural arrows $d$ and $e$, that is,

$$
\begin{aligned}
& \left.q d=d: q_{A} \rightarrow\left[q_{B}, q_{A} \otimes q_{B}\right]=q_{[B, A \otimes B}\right], \\
& q e=e: q_{[A B] \otimes A}=\left[q_{A} q_{B}\right] \otimes q_{A} \rightarrow q_{B} \text {. }
\end{aligned}
$$

In any monoidal closed category we have the relations

$$
\begin{aligned}
l & =e(j \otimes 1): I \otimes A \rightarrow[A A] \otimes A \rightarrow A, \\
r & =e(i \otimes 1): A \otimes I \rightarrow[I A] \otimes I \rightarrow A, \\
w & =[d 1] L:[A \otimes B, C] \rightarrow[[B, A \otimes B], B, C] \rightarrow[A, B, C],
\end{aligned}
$$

and they prove that the functor $q$ preserves the natural arrows $l, r$ and $w$.

Finally the relation,

$$
\begin{aligned}
a & =e(w \otimes 1)([1 e] e \otimes 1): A \otimes(B \otimes C) \rightarrow[B, A \otimes B] \otimes(B \otimes C) \\
& \rightarrow[B, C,(A \otimes B) \otimes C] \otimes(B \otimes C) \\
& \rightarrow[B \otimes C,(A \otimes B) \otimes C] \otimes(B \otimes C) \rightarrow(A \otimes B) \otimes C,
\end{aligned}
$$

proves that the functor $q$ preserves $a$. Thus we have proved the following proposition:

Proposition 4. The functor $q$ preserves any monoidal closed structure which may exist in $\mathbf{V}$. 


\section{REFERENCES}

1. J. Bénæbou, Catégories avec multiplication, C. R. Acad. Sci. Paris 256 (1963), 1887-1890. MR 26 \#6225.

2. B. Day, Construction of biclosed categories, Thesis, University of South Wales, 1970.

3. , On closed categories of functors, Reports of the Midwest Category Seminar IV, Lecture Notes in Math., vol. 137, Springer-Verlag, Berlin and New York, 1970, pp. 1-38. MR 42 \#7733.

4. S. Eilenberg and G. M. Kelly, Closed categories, Proc. Conf. Categorical Algebra (La Jolla, Calif., 1965), Springer-Verlag, Berlin and New York, 1966, pp. 421-562. MR 37 \# 1432.

5. _ A generalization of the functorial calculus, J. Algebra 3 (1966), 366-375. MR 32 \#7618.

6. G. M. Kelly, On Mac Lane's conditions for coherence of natural associativities, commutatives, etc., J. Algebra 1 (1964), 397-402. MR 32 \# 132.

7. G. M. Kelly and S. Mac Lane, Coherence in closed categories, J. Pure Appl. Algebra 1 (1971), 97-140. MR 44 \#278.

8. S. Mac Lane. Natural associativity and commutativity, Rice Univ. Studies 49 (1963), 28-46. MR 30 \# 1160.

9. , Categories for the working mathematician, Springer-Verlag, Berlin and New York, 1971.

Department of Mathematics, University of Puerto Rico, Mayaguez, Puerto Rico 00708 